\title{
Expression of HIF-1 regulated proteins vascular endothelial growth factor, carbonic anhydrase IX and hypoxia inducible gene 2 in hemangioblastomas
}

\author{
Mei Li, Jie Song, Peter Pytel \\ Department of Pathology, University of Chicago, Chicago, USA
}

Folia Neuropathol 2014; 52 (3): 234-242

DOI: $10.5114 / f n .2014 .45564$

\begin{abstract}
Introduction: Hemangioblastomas occur as sporadic or as von Hippel-Lindau syndrome (VHL) associated tumors. In both settings, activation of the VHL-HIF-1 (hypoxia induced factor) pathway is thought to be important in tumor biology.

Material and methods: We performed immunohistochemical studies on 23 hemangioblastomas, 13 meningiomas and 4 hemangiopericytomas to evaluate expression of the VHL-HIF-1 regulated proteins vascular endothelial growth factor (VEGF), carbonic anhydrase IX (CAIX) and hypoxia inducible gene 2 (HIG-2).

Results: Hemangioblastomas showed significantly higher expression of CAIX and VEGF than the other tested tumors. They showed strong membranous expression of CAIX in a pattern identical to that observed in clear cell renal cell carcinomas (CCRCC). Interestingly, hemangioblastomas lacked significant reactivity for HIG-2.

Conclusions: The VHL-HIF-1 regulated genes VEGF and CAIX are expressed in hemangioblastomas but significant HIG-2 expression is not observed. Carbonic anhydrase IX staining in particular may be a helpful marker of hemangioblastomas but does not aid in the distinction from CCRCC.
\end{abstract}

Key words: hemangioblastoma, von Hippel-Lindau, carbonic anhydrase IX.

\section{Introduction}

Hemangioblastomas are highly vascular tumors. Their two main components are prominent vascular channels and the so-called stromal cells. The latter are regarded as the true lesional cells but are of unclear histogenesis [11,29]. Common anatomic sites include the cerebellum (over $60 \%$ to $75 \%$ ), spinal cord and brainstem [3,7,21].

Hemangioblastomas can be associated with the autosomal dominant familial tumor syndrome von
Hippel-Lindau syndrome (VHL) or they can be sporadic [3,29].

Von Hippel-Lindau syndrome is a hereditary cancer syndrome. Affected patients have deactivating mutations of the VHL gene. This leads to an increased risk of developing a number of tumors including hemangioblastoma, clear cell renal cell carcinoma (CCRCC) and endolymphatic sac tumors $[10,21]$. Clear cell renal cell carcinomas are the leading cause of death in VHL patients [29]. Overall, some $20 \%$ to $30 \%$ of hemangioblastoma cases 
may be VHL syndrome associated [7]. Sporadic hemangioblastomas and sporadic CCRCC also commonly show bi-allelic VHL gene inactivation $[15,16,23,29]$.

The $\mathrm{VHL}$ gene product $\mathrm{pVHL}$ is a master regulator of HIF- $1 \alpha$ (hypoxia inducible factor-1 alpha). HIF-1 is a ubiquitously expressed heterodimeric basic-helix-loop-helix transcription factor composed of the highly unstable HIF- $1 \alpha$ and the stable HIF- $1 \beta$ subunit [16]. HIF-1 $\alpha$ is the main regulatory subunit. It helps to orchestrate the cellular response to hypoxic conditions. $\mathrm{PVHL}$, elongin $\mathrm{B}$ and elongin $\mathrm{C}$ form the VBC complex that is a key regulator of the HIF-1 $\alpha$ pathway [23]. Under normoxic conditions pVHL and the VBC complex direct HIF-1 $\alpha$ for polyubiquitination and degradation. The oxygen dependent nature of this reaction results from the fact that $\mathrm{pVHL}$ only recognizes HIF- $1 \alpha$ after oxygen-level sensitive hydroxylation of HIF-1 $\alpha$. Deactivating VHL mutations result in the abnormal accumulation of HIF-1 $\alpha$ and the subsequent over-expression of its downstream targets [15]. These target genes include carbonic anhydrase IX (CAIX), vascular endothelial growth factor (VEGF), erythropoietin, glucose transporters and glycolytic enzymes $[26,29]$.

Carbonic anhydrase IX (CAIX) is an isoenzyme of the $\alpha$-carbonic anhydrase family, which regulates intra- and extracellular $\mathrm{pH}$ by the reversible hydration of $\mathrm{CO}_{2}$ to form $\mathrm{HCO}^{3-}$ and protons [4]. Hypoxia is the main mechanism to induce expression of CAIX. Transcriptional activation of CAIX by hypoxia is mediated by HIF-1 via binding to the hypoxia-responsive element (HRE). In physiological conditions various normal tissues express CAIX at very low levels. It is overexpressed in CCRCC and under hypoxic conditions such as tissues around areas of necrosis. VHL-HIF-CAIX and VHL-HIF-VEGF pathways are thought to be important in the tumorigenesis of CCRCC in patients with or without VHL syndrome $[5,12,13]$. In CCRCC pVHL inactivation is found in preneoplastic renal tissue, suggesting that it represents an early step in carcinogenesis [15] Induction of CAIX in tumor cells might contribute to an aggressive phenotype by promoting cell proliferation, invasion and acid tolerance [12]. Vortmeyer et al. looked at "tumorlet"-like microscopic spinal cord lesions in autopsy samples of VHL patients and were able to show activation of the VHL-HIF-CAIX pathway in this setting by immunohistochemical studies [27].
Strong uniform membranous expression of CAIX is characteristic of CCRCC and often used as a diagnostic marker. Hypoxia inducible gene-2 (HIG-2) is another downstream target upregulated by the VHL-HIF-1 pathway and has recently been described as another marker of CCRCC $[1,6,25]$. One study suggested that HIG-2 is a novel lipid droplet protein that can stimulate intracellular lipid accumulation [6].

In most cases hemangioblastoma is a diagnosis that can be made based solely on the H\&E stain. In some instances and especially with fixation or thermal artifacts, the morphologic appearance of hemangioblastomas may mimic that of metastatic CCRCC and some primary intracranial tumors such as certain meningioma variants $[22,24]$. The differential diagnosis between metastatic CCRCC and hemangioblastoma is particularly critical in VHL syndrome patients since they are at increased risk of developing both of these tumors. This is illustrated by reports of VHL patients who were found to have metastatic carcinoma within CNS hemangioblastomas $[8,14,19]$. A few markers have been suggested as helpful in the diagnosis of hemangioblastoma and in distinguishing hemangioblastoma from CCRCC. Inhibin-A is expressed in $87 \%$ to $100 \%$ of hemangioblastomas $[2,9,22,28]$. Pax2 and Pax8 were shown to be negative in hemangioblastomas but positive in the vast majority of CCRCC $[2,22]$. Aquaporin 1 was found in nearly $100 \%$ of hemangioblastomas but only $18 \%$ of CCRCC $[24,28]$. Additionally the stromal cells are also reported to express vimentin (100\%), CD56/NCAM (100\%), VEGF (100\%), S-100 (82\%), Ezrin (59\%), and CD99 (88\%). Up to $36 \%$ of hemangioblastomas may be positive for EMA and some are positive for GFAP $[11,28]$. CD10 has been reported to be expressed in $12 \%$ of hemangioblastomas [22].

In the present study we conducted immunohistochemical staining of hemangioblastomas, hemangiopericytomas, and meningiomas including clear cell meningioma, microcystic meningioma as well as angiomatous meningioma. The aim was to address the following hypotheses: (A) Hemangioblastomas may express HIF-1 regulated proteins such as CAIX, VEGF and HIG-2 in a pattern similar to other VHL syndrome associated tumors, particularly CCRCC. (B) These HIF-1 regulated proteins may be useful diagnostic markers for distinguishing hemangioblastomas from possible mimics. 


\section{Material and methods}

This study was approved by the Institutional Review Board of the University of Chicago Medical Center. A total of 41 intracranial tumors were collected from the archive of the Pathology Department at the University of Chicago Medical Center. These included 23 hemangioblastomas (3 with documented association with VHL syndrome), 14 meningiomas, and 4 hemangiopericytomas (Table I). Microcystic (3), angiomatous (3) and clear cell (2) variants of meningioma were included. All the tissues were fixed in $10 \%$ neutrally buffered formalin and paraffin embedded. Sections were cut at $4 \mu \mathrm{m}$ and processed in batches for immunohistochemical staining using monoclonal anti-CAIX (obtained from Novus Biologicals, Littleton, Colorado, USA), monoclonal anti-VEGF (Santa Cruz Biotechnology, Santa Cruz, California, USA) and anti-HIG 2 (Novocastra/Leica Microsystems, Leica Microsystems, Buffalo Grove, Illinois, USA) antibodies.

Immunohistochemical staining was performed according to standard protocols. Briefly, sections were first deparaffinized and rehydrated, followed by antigen retrieval by heating the sections in EDTA buffer at pH 9 for 15 minutes. Endogenous peroxidase activity was removed by incubating the sections with $3 \% \mathrm{H}_{2} \mathrm{O}_{2}$ in methanol for 5 minutes. Non-specific binding was minimized by incubation with Protein Block (DAKO, Carpinteria, CA) for 20 minutes. After that, the sections were incubated with the primary antibody for 1 hour, followed by the secondary antibody conjugated to a horseradish peroxidase-labeled polymer for 30 minutes. Slides were then developed with 3-30-diaminobenzidine chromogen and counterstained with hematoxylin.

The slides were reviewed and scored independently by two pathologists. Staining extent, staining intensity and subcellular localization were evaluated. The percentage of the tumor areas showing strong (3+), moderate (2+), weak (1+) or negative (0) staining respectively were recorded. The extent of staining was classified as diffuse $(\geq 80 \%$ area with positive staining) or focal (1-79\%) unless further specified.

The staining index was calculated as a single numerical value to summarize and compare the level of staining: the percentage of area with $3+$ staining was multiplied by 3 , the percentage of area showing
$2+$ staining was multiplied by 2 , and the percentage of area with $1+$ staining was multiplied by 1 . The sum of these values is the staining index of an individual case $[17,18]$.

Statistical analysis was performed using GraphPad Prism version 6.0 for MacOS X, GraphPad Software, La Jolla California USA. The Kruskal-Wallis test was used to determine whether the observed differences between meningiomas, hemangiopericytomas and hemangioblastomas were statistically significant. Post-test analysis was performed by Dunn's multiple comparison test. This statistical analysis was performed on two parts of the results: (1) the percentage of tumor area showing positive staining of any intensity and (2) the calculated staining index.

\section{Results}

The results of the performed immunohistochemical staining are summarized in Table I.

\section{Carbonic anhydrase IX staining}

Carbonic anhydrase IX expression was detected in all the examined hemangioblastomas (23/23) with a strong diffuse membranous staining pattern (Figs. 1A-B). This pattern is identical to that described in CCRCC. Carbonic anhydrase IX staining in hemangioblastomas was significantly stronger than in meningiomas and hemangiopericytomas (Kruskal-Wallis: $p<0.0001$ ), none of which exhibited any strong membranous staining (Figs. 2A-B). In addition to strong membranous staining all the hemangioblastomas also showed at least focal weak cytoplasmic staining.

In meningiomas of various subtypes, only weak cytoplasmic staining, either focal or diffuse, was observed. The tumor cells of microcystic meningiomas showed focal or diffuse weak cytoplasmic staining, particularly surrounding the microcystic spaces. Angiomatous meningiomas and meningiomas with clear cell features also showed diffuse weak cytoplasmic staining. None of the meningiomas exhibited any significant membranous labeling.

All the hemangiopericytomas showed weak to moderate cytoplasmic staining of CAIX. In one hemangiopericytoma, tumor cells surrounding an area of necrosis exhibited strong membranous staining in a pattern interpreted as being the result of regional hypoxia. 


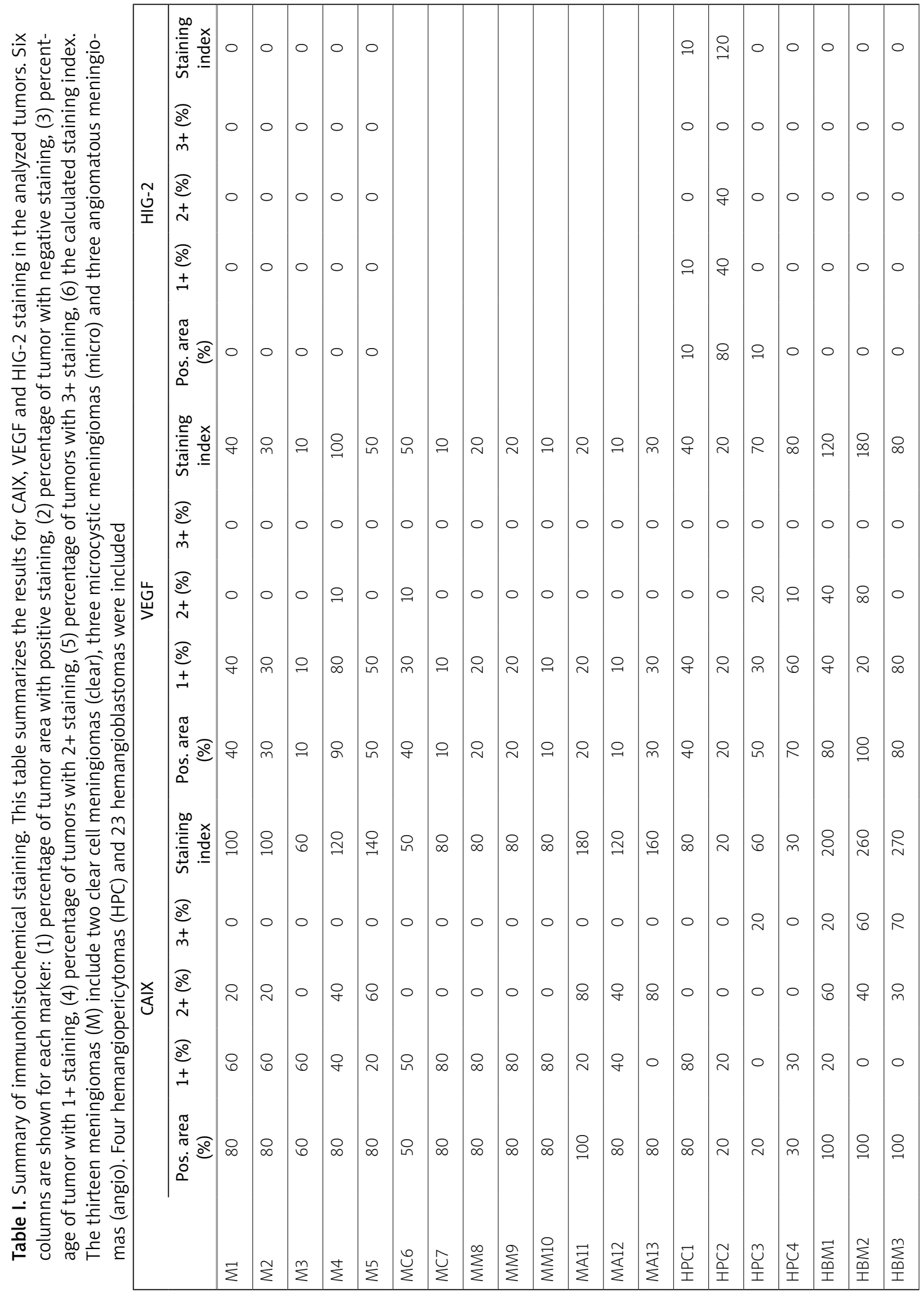




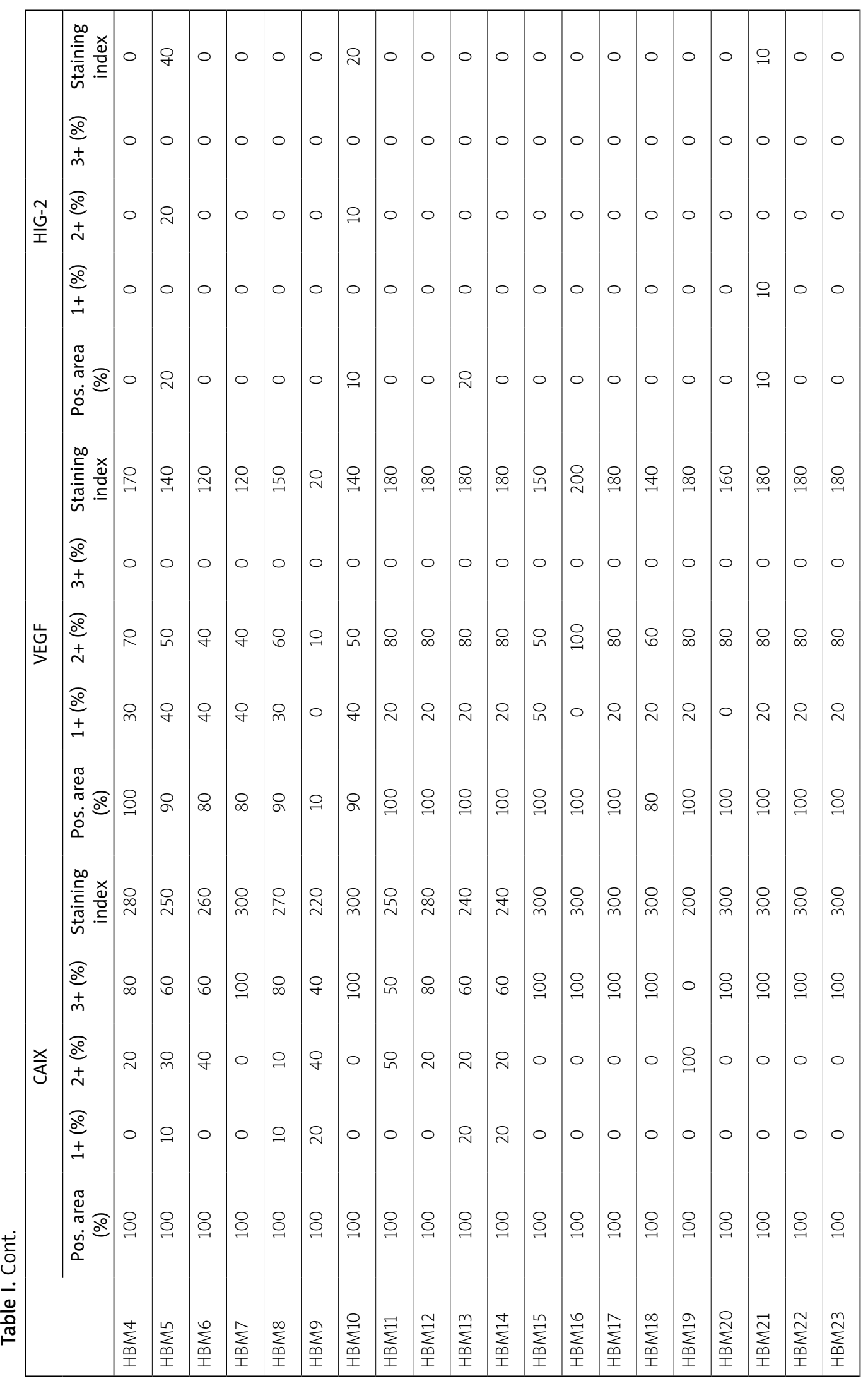



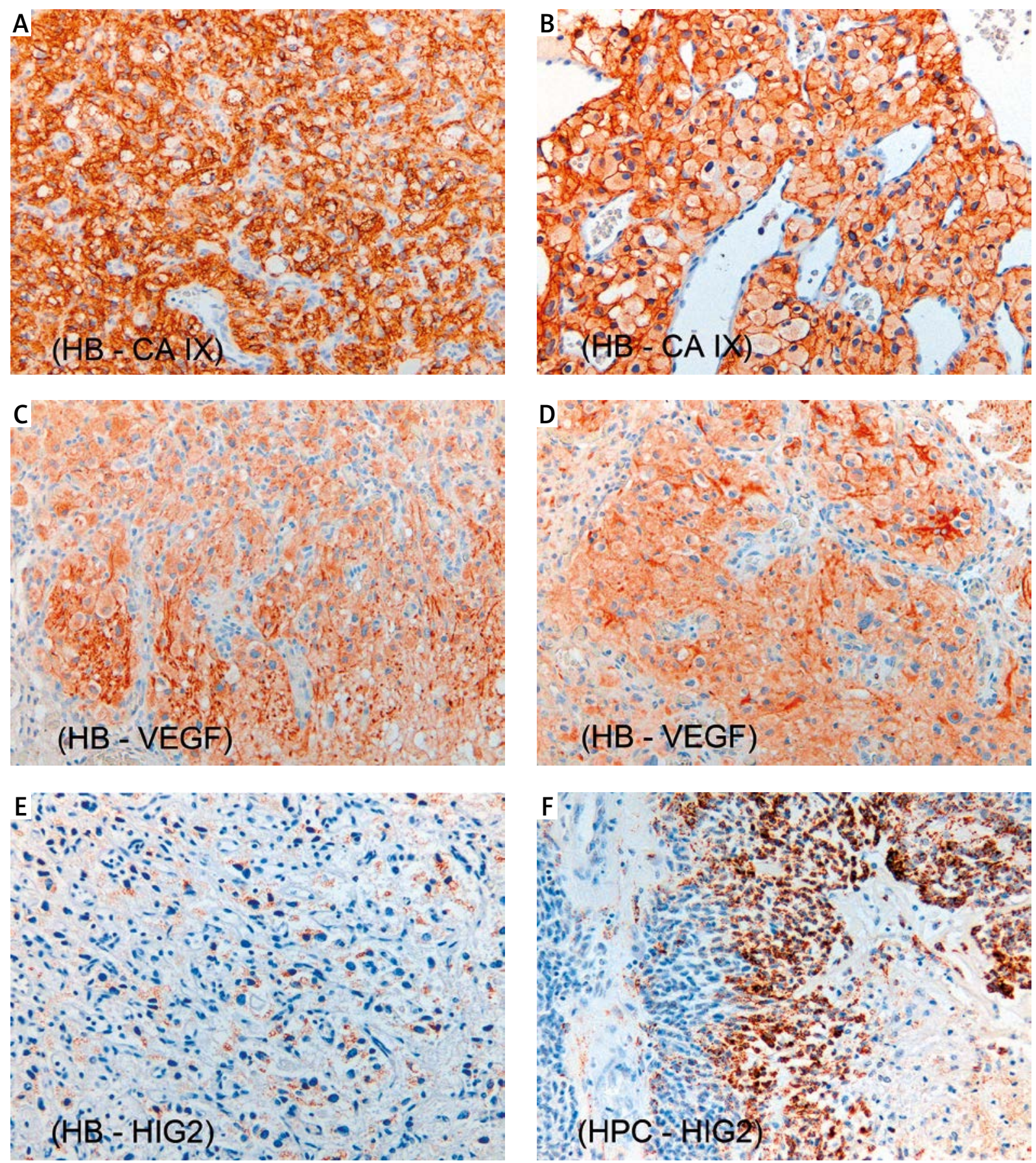

Fig. 1. Representative images of immunohistochemical staining. A) and B) illustrate staining in hemangioblastomas (HB). In general, these exhibited cytoplasmic staining of variable intensity and strong uniform cell membrane staining. C) and D) show two examples of VEGF staining in hemangioblastomas (HB). E) and F) illustrate the lack of significant HIG-2 staining in hemangioblastomas (E) and the focal distinct immunoreactivity in a hemangiopericytoma $(F)$ around an area of necrosis. 

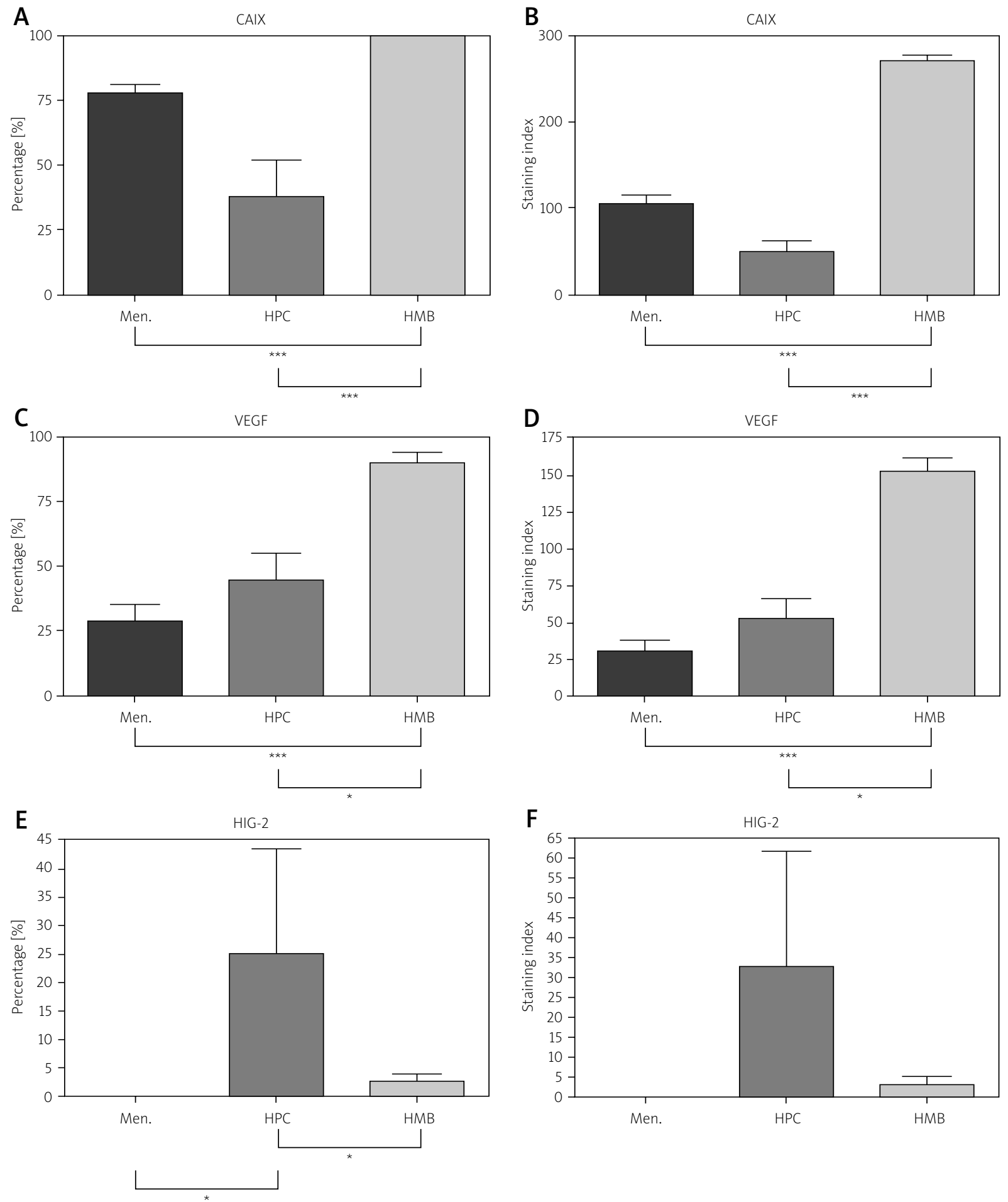

Fig. 2. The graphic representation of the results for CAIX (A and B), VEGF (C and D) and HIG-2 (E and F) include the comparison of two separate features: (1) the percentage of tumor labeled positive for a given marker irrespective of the intensity of staining ( $A, C$ and $\mathbf{E})$ and the calculated staining index (B, D and $\mathbf{F})$. The differences illustrated in figures A, B, C and D were all statistically significant with a $p$ value of $<0.0001$ in the Kruskal-Wallis test. Brackets at the bottom of individual graphs are used to indicate statistical significance in the pairwise comparison ( ${ }^{*}$ indicates a $p$ value of $0.05,{ }^{* * *}$ indicates a $p$ value of $<0.001$ ). 


\section{Vascular endothelial growth factor staining}

All the hemangioblastomas (23/23) showed cytoplasmic staining (Figs. 1C-D). Most hemangioblastomas (17/23) demonstrated diffuse moderate cytoplasmic staining. Overall, hemangioblastomas exhibited significantly stronger VEGF immunoreactivity than the other two types of tumor (Kruskal-Wallis: $p<0.0001$; see Figs. 2C-D). Most meningiomas (12/14) showed scattered, very focal $(<30 \%$ area) weak cytoplasmic staining.

\section{Hypoxia inducible gene 2 staining}

Hypoxia inducible gene 2 staining was low in all three types of tumor. Overall, hemangiopericytomas showed slightly higher expression of HIG-2 than hemangioblastomas or meningiomas (Figs. 1E-F). Some hemangiopericytomas showed strong expression of HIG-2 in perinecrotic areas (Fig. 1F). This was interpreted as an internal positive control that supports the adequacy of the stain.

\section{Discussion}

It has long been recognized that hemangioblastomas can be associated with polycythemia and with intratumoral extramedullary hematopoiesis [23]. These features have been attributed to erythropoietin overproduction - a sign of VHL-HIF-1 signaling pathway activation. Our study looked at the expression of other hypoxia induced markers. We hypothesized that these would be expressed in hemangioblastomas in analogy to findings in CCRCC and as a result of VHL-HIF-1 signaling pathway activation. Vascular endothelial growth factor expression in hemangioblastomas has been reported by Ishizawa [11]. We have seen similar results and found stronger VEGF expression in hemangioblastomas compared to meningiomas and hemangiopericytomas.

Proescholdt et al. conducted a study of CAIX expression in brain tumors of various type. They report strong CAIX staining in the few included cases of hemangioblastomas [20]. However, the staining pattern was not described and the potential diagnostic value was not discussed. We compared the expression of CAIX in hemangioblastomas with some of its potential mimickers. Similar to CCRCC, hemangioblastomas showed strong diffuse membranous expression of CAIX. Based on these results, CAIX cannot be relied on as a marker for establish- ing a diagnosis of CCRCC when hemangioblastoma is part of the differential diagnosis. Strong staining for CAIX with membranous accentuation is, however, a helpful diagnostic marker in distinguishing hemangioblastoma from other intracranial tumors. In the authors' opinion, CAIX provides more robust labeling of hemangioblastomas than other reported markers, including D2-40 and inhibin A.

Several HIF-1 inducible proteins such as erythropoietin, VEGF and CAIX are expressed in hemangioblastomas. In this context it is interesting to note that HIG-2 [1,6,25], as another hypoxia inducible and HIF-1 regulated protein, did not appear to be expressed in hemangioblastomas even though it has been shown to be a marker of CCRCC. This difference could point to differences in the detailed expression profile of hypoxia inducible genes between CCRCC and hemangioblastomas.

In summary, hemangioblastomas uniformly express VHL-HIF-1 regulated proteins including VEGF and CAIX. A strong membranous staining pattern for CAIX can be a helpful marker of hemangioblastoma in the distinction from other intracranial tumors but cannot be used to exclude the possibility of metastatic CCRCC.

\section{Disclosure}

Authors report no conflict of interest.

\section{References}

1. Argani P, Hicks J, De Marzo AM, Albadine R, Illei PB, Ladanyi M, Reuter VE, Netto GJ. Xp11 translocation renal cell carcinoma (RCC): extended immunohistochemical profile emphasizing novel RCC markers. Am J Surg Pathol 2010; 34: 1295-1303.

2. Carney EM, Banerjee P, Ellis CL, Albadine R, Sharma R, Chaux AM, Burger PC, Netto GJ. PAX2(-)/PAX8(-)/inhibin A(+) immunoprofile in hemangioblastoma: A helpful combination in the differential diagnosis with metastatic clear cell renal cell carcinoma to the central nervous system. Am J Surg Pathol 2011; 35: 262-267.

3. Catapano D, Muscarella LA, Guarnieri V, Zelante L, D’Angelo VA, D’Agruma L. Hemangioblastomas of central nervous system: molecular genetic analysis and clinical management. Neurosurgery 2005; 56: 1215-1221.

4. Chegwidden WR, Dodgson SJ, Spencer IM. The roles of carbonic anhydrase in metabolism, cell growth and cancer in animals. EXS 2000; 90: 343-363.

5. Clark PE. The role of VHL in clear-cell renal cell carcinoma and its relation to targeted therapy. Kidney Int 2009; 76: 939-945.

6. Gimm T, Wiese M, Teschemacher B, Deggerich A, Schodel J, Knaup KX, Hackenbeck T, Hellerbrand C, Amann K, Wiesener MS, Honing S, Eckardt KU, Warnecke C. Hypoxia-inducible protein 2 
is a novel lipid droplet protein and a specific target gene of hypoxia-inducible factor-1. FASEB J 2010; 24: 4443-4458.

7. Glasker S. Central nervous system manifestations in VHL: genetics, pathology and clinical phenotypic features. Fam Cancer 2005; 4: 37-42.

8. Hamazaki S, Nakashima H, Matsumoto K, Taguchi K, Okada S. Metastasis of renal cell carcinoma to central nervous system hemangioblastoma in two patients with von Hippel-Lindau disease. Pathol Int 2001; 51: 948-953.

9. Hoang MP, Amirkhan RH. Inhibin alpha distinguishes hemangioblastoma from clear cell renal cell carcinoma. Am J Surg Pathol 2003; 27: 1152-1156.

10. Horiguchi H, Sano T, Toi H, Kageji T, Hirokawa M, Nagahiro S. Endolymphatic sac tumor associated with a von Hippel-Lindau disease patient: an immunohistochemical study. Mod Pathol 2001; 14: 727-732.

11. Ishizawa K, Komori T, Hirose T. Stromal cells in hemangioblastoma: neuroectodermal differentiation and morphological similarities to ependymoma. Pathol Int 2005; 55: 377-385.

12. Ivanov S, Liao SY, Ivanova A, Danilkovitch-Miagkova A, Tarasova N, Weirich G, Merrill MJ, Proescholdt MA, Oldfield EH, Lee J, Zavada J, Waheed A, Sly W, Lerman MI, Stanbridge EJ. Expression of hypoxia-inducible cell-surface transmembrane carbonic anhydrases in human cancer. Am J Pathol 2001; 158: 905-919.

13. Ivanov SV, Kuzmin I, Wei MH, Pack S, Geil L, Johnson BE, Stanbridge EJ, Lerman MI. Down-regulation of transmembrane carbonic anhydrases in renal cell carcinoma cell lines by wild-type von Hippel-Lindau transgenes. Proc Natl Acad Sci U S A 1998; 95: 12596-12601.

14. Jarrell ST, Vortmeyer AO, Linehan WM, Oldfield EH, Lonser RR. Metastases to hemangioblastomas in von Hippel-Lindau disease. J Neurosurg 2006; 105: 256-263.

15. Kaelin WG. The von Hippel-Lindau tumor suppressor protein: roles in cancer and oxygen sensing. Cold Spring Harb Symp Quant Biol 2005; 70: 159-166.

16. Kim WY, Kaelin WG. Role of VHL gene mutation in human cancer. J Clin Oncol 2004; 22: 4991-5004.

17. Luukkaa H, Klemi P, Hirsimaki P, Vahlberg T, Kivisaari A, Kahari VM, Grenman R. Matrix metalloproteinase (MMP)-1, -9 and -13 as prognostic factors in salivary gland cancer. Acta Otolaryngol 2008; 128: 482-490.

18. Menczer J, Schreiber L, Sukmanov O, Kravtsov V, Berger E, Golan A, Levy T. COX-2 expression in uterine carcinosarcoma. Acta Obstet Gynecol Scand 2010; 89: 120-125.

19. Polydorides AD, Rosenblum MK, Edgar MA. Metastatic renal cell carcinoma to hemangioblastoma in von Hippel-Lindau disease. Arch Pathol Lab Med 2007; 131: 641-645.

20. Proescholdt MA, Mayer C, Kubitza M, Schubert T, Liao SY, Stanbridge EJ, Ivanov S, Oldfield EH, Brawanski A, Merrill MJ. Expression of hypoxia-inducible carbonic anhydrases in brain tumors. Neuro Oncol 2005; 7: 465-475.

21. Richard S, David P, Marsot-Dupuch K, Giraud S, Beroud C, RescheF. Central nervous system hemangioblastomas, endolymphatic sac tumors, and von Hippel-Lindau disease. Neurosurg Rev 2000; 23: 1-22.

22. Rivera AL, Takei H, Zhai J, Shen SS, Ro JY, Powell SZ. Useful immunohistochemical markers in differentiating hemangio- blastoma versus metastatic renal cell carcinoma. Neuropathology 2010; 30: 580-585.

23. Shuin T, Yamasaki I, Tamura K, Okuda H, Furihata M, Ashida S. Von Hippel-Lindau disease: molecular pathological basis, clinical criteria, genetic testing, clinical features of tumors and treatment. Jpn J Clin Oncol 2006; 36: 337-343.

24. Takei H, Powell SZ. Novel immunohistochemical markers in the diagnosis of nonglial tumors of nervous system. Adv Anat Pathol 2010; 17: 150-153.

25. Togashi A, Katagiri T, Ashida S, Fujioka T, Maruyama O, Wakumoto Y, Sakamoto Y, Fujime M, Kawachi Y, Shuin T, Nakamura Y. Hypoxia-inducible protein 2 (HIG2), a novel diagnostic marker for renal cell carcinoma and potential target for molecular therapy. Cancer Res 2005; 65: 4817-4826.

26. Tostain J, Li G, Gentil-Perret A, Gigante M. Carbonic anhydrase 9 in clear cell renal cell carcinoma: a marker for diagnosis, prognosis and treatment. Eur J Cancer 2010; 46: 3141-3148.

27. Vortmeyer AO, Tran MG, Zeng W, Glasker S, Riley C, Tsokos M, Ikejiri B, Merrill MJ, Raffeld M, Zhuang Z, Lonser RR, Maxwell PH, Oldfield EH. Evolution of VHL tumourigenesis in nerve root tissue. J Pathol 2006; 210: 374-382.

28. Weinbreck N, Marie B, Bressenot A, Montagne K, Joud A, Baumann C, Klein O, Vignaud JM. Immunohistochemical markers to distinguish between hemangioblastoma and metastatic clearcell renal cell carcinoma in the brain: utility of aquaporin1 combined with cytokeratin AE1/AE3 immunostaining. Am J Surg Pathol 2008; 32: 1051-1059.

29. Zagzag D, Krishnamachary B, Yee H, Okuyama H, Chiriboga L, Ali MA, Melamed J, Semenza GL. Stromal cell-derived factor-1alpha and CXCR4 expression in hemangioblastoma and clear cell-renal cell carcinoma: von Hippel-Lindau loss-of-function induces expression of a ligand and its receptor. Cancer Res 2005; 65: 6178-6188. 